\title{
O QUE NÃO É E O QUE PODE VIR A SER COMPORTAMENTO
}

\author{
BEHAVIOR, WHAT IT IS NOT AND WHAT IT MIGHT BE \\ JoÃo CLAUdio TODOROV \\ UNIVERSIDADE dE BRASÍLIA, BRASIL \\ MARcelo Borges Henriques \\ Universidade de Brasília e Universidade FEderal de GoIás, BrasiL
}

\section{RESUMO}

O termo "comportamento" pode ser utilizado em diferentes contextos com diferentes significados, mas na Análise do Comportamento ele às vezes é utilizado como sinônimo da palavra "interação". Interações, no plural, são o objeto de estudo da Psicologia e, na Análise do Comportamento, são estudadas por meio da análise de contingências. Contingências não se restringem a operantes, mas quando a literatura na área se refere à definição de comportamento, ela normalmente o faz descrevendo a contingência operante: comportamento em interação com o ambiente.

Palavras-chave: comportamento; contingências; análise do comportamento.

\begin{abstract}
The word "behavior" can be used in different contexts, with different meanings, for living organisms or inanimate things, but in Behavior Analysis sometimes it is used as synonymous of the word "interaction". Interactions, in the plural form, are the subject of Psychology, and, in the special case of behavior analysis, are studied through analysis of contingencies. Contingencies are not restricted to operants, but the literature has used the word behavior as synonymous of the operant contingency: behavior in interaction with the environment.

Keywords: behavior; contingencies; behavior analysis.
\end{abstract}

João Cláudio Todorov é Bolsista de Produtividade Científica 1D do CNPq. Partes deste trabalho foram publicadas no blog jctodorov.blogspot.com.br.

Endereço de contato, todorov@unb.br 
Os verbos e expressões que designam o que um indivíduo fez, faz ou fará são genericamente denominados comportamentos. Comportamento é empregado com diferentes sentidos em várias disciplinas científicas e na linguagem ordinária. Buscar uma única definição ou a essência do conceito talvez seja tão problemático quanto o esforço para definir consciência (Low, Reiss, Edelman, Van Swinderen, \& Koch, 2012) ou reflexo (Prochazka, Clarac, Loeb, Rothwell, \& Wolpaw, 2000). Neste trabalho nos preocupamos com a definição "usada" na Análise do Comportamento e esclarecemos uma distinção conceitual entre os termos comportamento e contingência comportamental (seja respondente, operante ou cultural).

\section{COMPORTAMENTO COMO OBJETO DE ESTUDO DA ANÁLISE DO COMPORTAMENTO}

Definir comportamento fez parte do empenho do behaviorismo em diferenciar-se da psicologia tradicional. Tanto quanto Watson, Skinner se viu às voltas com a psicologia dominante da época, para a qual as ações dos indivíduos eram indícios de processos subjacentes. Advogar o comportamento como um objeto de estudo per se objetivou destacar que aquilo que os organismos fazem não é um indício de outros processos, se não, da interação entre o comportamento e os eventos ambientais circunscritos (Moore, 2011).

Moore (2008) define comportamento como interação entre organismo e ambiente:

The sense of behavior that is relevant to radical behaviorism is that it is an interaction between organism and environment that has particular properties as a result of certain functional relations that obtain between the features of the behavior and features of the environment (p. 68).

Concordamos que a psicologia é o estudo de interações, mas discordamos que comportamento seja interação. As abordagens, áreas, teorias, quando vistas sob a ótica da Análise do Comportamento, estudam interações que envolvem comportamento e ambiente, seja este interno (biológico ou histórico) ou externo (físico ou social) ao organismo. A psicologia é uma ponte que liga as ciências biológicas, de um lado, e as ciências sociais, de outro; as fronteiras não são delimitadas (Todorov, 1989, 2006, 2010).

As interações que são estudadas pela Análise do Comportamento são relações condicionais entre comportamento e os eventos ambientais. Essas interações são estudadas, preditas e controladas por meio de um instrumento conceitual denominado de contingência. As contingências descrevem relações funcionais de tal sorte que o conhecimento apurado da dinâmica interacional nos permite programar novas interações. Em um artigo publicado em 1966, após a fundação da Divisão 25 da American Psychological Association, Skinner se preocupou em distinguir o programa de pesquisas em Análise Experimental do Comportamento diferenciando-o dos demais. Neste artigo Skinner caracterizou a principal unidade de medida e de análise da ciência do comportamento. O primeiro ponto abordado por Skinner foi a descrição da variável dependente. Para Skinner (1966) uma ciência do comportamento deveria ser capaz de registrar ocorrências de instâncias repetidas de desempenho, uma dificuldade imposta ao cientista uma vez que certos desempenhos não obedecem uma estereotipia topográfica e, portanto, operacional. A solução relatada pelo autor foi definir as ocorrências a partir da observação do efeito da ação.

$\mathrm{O}$ efeito de respostas individuais (i.e., instâncias) é o dado fundamental pelo qual o analista do comportamento pode observar classes de respostas; então, poder-se-ia dizer que a sua variável dependente é composta de respostas (i.e., com topografias que podem variar em diferentes dimensões) com efeitos comuns (e.g., o fechamento de um circuito eletrônico dado uma pressão a barra por um rato). À medida que o comportamento interage com o ambiente algumas dimensões de instâncias de respostas geram o efeito necessário para a produção de consequências, outras dimensões não. As consequências da interação retroagem sobre as dimensões que produzem o efeito, selecionando não só respostas, mas o efeito produzido, ou seja, comportamento. A passagem anterior é descrita por Todorov (2012):

Há dois aspectos do ambiente a serem considerados: (a) um efeito sobre o ambiente resultante da resposta e (b) alguma consequência que depende desse efeito. (. . .) A análise do comportamento operante estuda comportamento, definido pelo efeito (a) com o ambiente (consequências do tipo[b]) . . . Alguma confusão pode ocorrer quando, no operante, não 
separamos o efeito que nos ajuda a definir a resposta (e.g., o fechamento do circuito elétrico provocado pela pressão à barra) da consequência (p. 34).

Estudamos e intervimos nos parâmetros das interações (i.e., o reforço pode ser contingente à taxa de resposta, intensidade ou padrões específicos; o reforço pode variar em frequência e/ou magnitude; o respondente pode variar em magnitude a depender do intensidade do eliciador; etc.) (Weingarten \& Mechner, 1966). Desse modo, a unidade de análise do programa analítico comportamental seria a relação entre classes de respostas/efeitos e classes de estímulos, e o principal instrumento de apreciação seria a contingência.

Comportamento não está no organismo, é parte daquilo que o organismo faz (Skinner, 1938) e, como tal, não pode ser a interação entre um e outro evento. Não estudamos organismos como um todo, estudamos alterações em ações, não em organismos (Lee, 1992). O conceito de comportamento deve se diferenciar do conceito de contingência. No primeiro uso importa o conjunto de ações de um indivíduo observáveis objetivamente, não importando nenhuma referência em relação a eventos antecedentes ou consequentes. Para se definir um comportamento basta referência aos efeitos ambientais da ação (Skinner, 1938). Já no segundo termo, são necessários os referentes ambientais dos quais um conjunto de propriedades (efeitos) é função; neste sentido, classes de comportamento são conjuntos funcionais que agrupam relações condicionais entre comportamentoambiente.

\section{RESPOSTA (OPERANTE) É SINÔNIMO DE COMPORTAMENTO (OPERANTE)?}

Não, não é. Comportamento é classe, resposta é instância; respostas são definidas pela observação instantânea de um efeito, comportamento pela observação recorrente de efeito comum a todas as instâncias. Resposta é uma instância da classe comportamento. Comportamento é a variável dependente, é o que se pretende explicar e/ou entender, da histeria estudada por Freud ao movimento das fibras musculares de Hefferline, Keenan e Harford (1959), ou neurônios motores de Chapin, Moxon, Markowitz e Nicolelis (1999).
Variam a linguagem, o ponto no contínuo biológicosocial, o interesse prático ou teórico, mas sempre a psicologia trata do comportamento de indivíduos, chamados de pessoas quando humanos. Se para a Análise do Comportamento todos esses exemplos são exemplos de comportamento, como definir comportamento? "The study of behavior, psychotic or otherwise, remains securely in the company of the natural science as long as we take as our subject matter the observable activity of the organism. . ." (Skinner, 1999, p. 306)

Ao escrever o artigo "Sobre uma definição de comportamento", Todorov (2012) mostrou como uma definição em voga é inadequada. Ao definir comportamento como a interação organismo-ambiente autores passam a escrever sobre comportamento como parte da interação organismo-ambiente:

Uma resposta enganosa e tentadoramente simples é dizer que comportamento é a interação entre organismo e ambiente. Por exemplo, Moore (2008) afirma que o comportamento tem certas propriedades que resultam de certas relações funcionais entre aspectos do comportamento e aspectos do ambiente. Substituindo a palavra comportamento pela definição acima, temos a seguinte frase sem sentido: A interação entre organismo e ambiente tem certas propriedades que resultam de certas relações funcionais entre aspectos da interação entre organismo e ambiente e aspectos do ambiente (Todorov, 2012, p. 33).

A possível explicação para a prevalência dessa definição pode estar na forma pela qual Skinner (1953) definiu a contingência operante:

The unit of a predictive science is (...) not a response but a class of responses. The word "operant" will be used to describe this class. The term emphasizes the fact that behavior operates upon the environment to generate consequences. The consequences define the properties with respect to which responses are called similar [ênfase adicionada] (. . .) an operant is defined by an effect, which may be specified in physical terms ... (p. 65)

Os operantes são definidos pelas relações condicionais com as consequências; a confusão surge quando o termo comportamento se confunde com a contingência operante. Quando Skinner (1957) diz que os homens agem sobre o mundo (comportamento), 
modificam-no (efeito) e são modificados por alterações no ambiente (consequência), está falando de operantes. A confusão pode se justificar pelo extensivo estudo e discussão da contingência operante dentro da Análise do Comportamento somado com a justaposição da definição de Skinner de reflexo: Correlação estímulo-resposta (Skinner, 1935).

Comportamento e efeito são tão inseparáveis quanto estímulo e resposta no reflexo, mas nos dois casos o que interessa para a psicologia é a relação arbitrária com o ambiente. No respondente, como um estímulo neutro antecedente passa a eliciar a resposta; no operante, como uma consequência passa a modificar um comportamento.

Organismos não vivem no vácuo. Não é possível ocorrer qualquer ação do organismo sem alguma relação com o ambiente, externo ou interno ao organismo. Isso é elementar. Por isso, dizemos que comportamento não é coisa; é processo. Qualquer instância de comportamento tem início, meio e fim. Para a psicologia, essa é sempre a nossa variável dependente, independentemente da topografia ou do tipo de relação com o ambiente que definem essa variável dependente (e.g., respondentes e afins, operantes, padrões fixos de resposta, etc.). Variáveis independentes são variações no ambiente que afetam a ocorrência desses comportamentos, seja como antecedentes (no respondente e afins) ou consequentes (no operante e afins) (Todorov, 2012, p. 34).

Comportamento é muito mais que o operante (operante é uma das formas de análise de comportamento). Sua definição como a interação da qual faz parte parece vir de uma confusão entre efeito e consequência. Um mero aceno de mão tem como efeito o deslocamento do ar, alguém acenando de volta é a consequência. Falar "bom dia” tem como efeito a produção de ondas sonoras, alguém responder "bom dia" é a consequência. Na linguagem leiga identificamos comportamentos por seus efeitos no ambiente e explicamos esses comportamentos por suas consequências. No comportamento reflexo a resposta não pode ser definida sem o estímulo eliciador, nem o estímulo sem a resposta eliciada. $\mathrm{O}$ equivalente no comportamento operante é a relação comportamento-efeito, não a relação comportamentoconsequência.
Sobre efeito e consequência: Correr é comportamento, certo? É definido pelo deslocamento rápido no espaço. Não precisamos saber a causa para dizer que correr é comportamento. Já fuga, exercício, alcançar o ônibus são operantes definidos pela função da corrida. Dizemos que a criança está gritando pela intensidade do som produzido (efeito); dizemos que é birra pela consequência - o grito cessa quando a mãe dá atenção à criança. A consequência qualifica (birra) uma definição mais ampla (gritar). O grito é o comportamento, o qual é qualificado pela interação antecedente-comportamento-consequência. Exemplos semelhantes são milhares: Girar a maçaneta (comportamento), abrir a porta (operante); correr (comportamento), pegar o ônibus (operante); correr (comportamento), fugir da chuva (operante), falar (comportamento), mentira (operante).

\section{REFERÊNCIAS}

Chapin, J. K., Moxon, K. A., Markowitz, R. S., \& Nicolelis, M. A. (1999). Real-time control of a robot arm using simultaneously recorded neurons in the motor cortex. Nature Neuroscience, 2, 664-670.

Hefferline, R. F., Keenan, B., \& Harford, R. A. (1959). Escape and avoidance conditioning in human subjects without their observation of the response. Science, 130, 1338-1339.

Lee, V. L. (1992). Transdermal interpretation of the subject matter of behavior analysis. American Psychologist, 47, 1337-1343.

Low, P., Panksepp, J., Reiss, D., Edelman, D., Van Swinderen, B., Low, P., \& Koch, C. (2012). The Cambridge declaration on consciousness. Paper presentedatthe Francis CrickMemorial Conference on Consciousness in Human and Non-human Animals. University of Cambridge, Cambridge, England. Retirado de http://fcmconference. org/ img/CambridgeDeclarationOnConsciousness. $p d f$.

Moore, J. (2008). Conceptual foundations of radical behaviorism. Cornwall-on-Hudson: Sloan Publishing.

Moore, J. (2011). Behaviorism. The Psychological Record, 61, 449- 464. 
Prochazka, A., Clarac, F., Loeb, G. E., Rothwell, J. C., \& Wolpaw, J. R. (2000). What do reflex and voluntary mean? Modern views on an ancient debate. Experimental Brain Research, 130, 417-432.

Skinner, B. F. (1935). The generic nature of the concepts of stimulus and response. The Journal of General Psychology, 12, 40-65.

Skinner, B. F. (1938). The behavior of organisms: An experimental analysis. Acton: Copley.

Skinner, B. F. (1957). Verbal behavior. New York: Appleton-Century-Crofts.

Skinner, B. F. (1966). What is the experimental analysis of behavior? Journal of the Experimental Analysis of Behavior, 9, 213-218.

Skinner, B. F. (1999). Cumulative record. Cambridge: The B. F. Skinner Foundation.

Todorov, J. C. (1989). A psicologia como o estudo de interações. Psicologia: Teoria e Pesquisa, 5, 325347.

Todorov, J. C. (2006). The metacontingency as a conceptual tool. Behavior and Social Issues, 15, 92-94.

Todorov, J. C. (2010). Schedules of cultural selection: Comments on "Emergence and Metacontingency". Behavior and Social Issues, 19, 86-89.

Todorov, J. C. (2012). Sobre uma definição de comportamento. Perspectivas em Análise do Comportamento, 3, 32-37.

Weingarten,K., \& Mechner,F.(1966). The contingency as an independent variable of social interaction. Em T. Verhave, (Org.). The experimental analysis of behavior; selected readings (pp. 447-470). New York, Appleton-Century-Crofts. 